\section{Abscisic Acid Effect on Improving Horse Chestnut Secondary Somatic Embryogenesis}

\author{
Dušica Ćalić1, Nina Devrnja, and Jelena Milojević \\ Institute for Biological Research "Siniša Stanković," University of Belgrade, \\ Despot Stefan Boulevard 142, 11060 Belgrade, Serbia
}

\author{
Igor Kostić \\ Institute for Multidisciplinary Research, University of Belgrade, Kneza \\ Višeslava 1, 11000 Belgrade, Serbia
}

\author{
Dušica Janošević \\ Faculty of Biology, University of Belgrade, Takovska 43, 11060 Belgrade, \\ Serbia \\ Snežana Budimir and Snežana Zdravković-Korać \\ Institute for Biological Research "Siniša Stanković," University of Belgrade, \\ Despot Stefan Boulevard 142, 11060 Belgrade, Serbia
}

Additional index words. abscisic acid, Aesculus hyppocastanum, anther culture, microspore culture, secondary somatic embryogenesis

\begin{abstract}
The effect of abscisic acid on the development of primary androgenic embryo and secondary somatic embryogenesis was investigated with the aim of improving multiplication rates and secondary somatic embryo quality in horse chestnut microspore and anther culture. The early embryo stage (globular) had a better response than late stages (heart, torpedo, and cotyledonary) in both types of cultures. Also, microspore culture had a high potential for mass secondary embryo production. The number of secondary somatic embryos was three times higher on hormone-free medium than on medium enriched with $0.01 \mathrm{mg} \cdot \mathrm{L}^{-1}$ abscisic acid. However, most of the embryos on hormone-free medium had abnormal morphology. For this reason, abscisic acid was added to the media to improve embryo quality. The morphology of abscisic acid treated embryos was better than abscisic acid non-treated embryos. The optimal abscisic acid concentration for secondary somatic embryo induction and production of high-quality embryos was $0.01 \mathrm{mg} \cdot \mathrm{L}^{-1}$. Overall, the effect of abscisic acid on the induction of secondary somatic embryogenesis and plant regeneration of androgenic embryos of this species may be helpful for the further synthesis of secondary metabolites in vitro and their application in the pharmaceutical industry.
\end{abstract}

Horse chestnut (Aesculus hyppocastanum L., family Hyppocastanaceae) is an important medical and horticultural plant. It is a relict species and endemic of the Balkan Peninsula. The horse chestnut is a highly adaptive species to polluted urban environments, but it is very susceptible to the leaf miner Cameraria ohridella. C. ohridella infection heavily damages many horse chestnut stands and it has spread from Macedonia, where it was first discovered through Serbia and into central Europe (Gilbert et al., 2005).

An active component of the seed is usually used for the treatment of peripheral vascular disorders (Bombardelli et al., 1996). Also, it is used in cosmetics as a remedy

\footnotetext{
Received for publication 31 May 2012. Accepted for publication 5 Oct. 2012.

This work is supported by the Ministry of Education and Science of the Republic of Serbia (grants No. 173015 and III 43010).

${ }^{1}$ To whom reprint requests should be addressed; e-mail calic@ibiss.bg.ac.rs.
}

against cellulitis (Matsuda et al., 1997). It has anti-inflammatory (Matsuda et al., 1997), antitumor, antiviral (Yang et al., 1999), and antioxidative (Wilkinson and Brown, 1999) effects. A triterpenic glycoside known as aescin is an active component of the horse chestnut seed extract. Cotyledons of seed embryos are the main source of those compounds. Horse chestnut seeds are the sole industrial source of aescin.

This raw material is available only for a few months during the year. Caldas and Machado (2004) found high levels of lead in leaves and seed extracts of horse chestnut. As a result of this, haploids, diploids (Ćalić-Dragosavac et al., 2010b) as well as secondary somatic embryos in vitro may be a contaminant-free alternative solution for aescin production.

Within the genus Aesculus, secondary somatic embryogenesis (SSE) induction has only been reported in horse chestnut (Ćalić et al., 2005a), red chestnut (Zdravković et al., 2008), and yellow bucket (Ćalić et al., 2005b).
These studies were specifically focused on SSE on primary horse chestnut androgenic embryos. Because of the low frequency of induction of SSE on media with $2.5,10$, and $20 \mathrm{mg} \cdot \mathrm{L}^{-1}$ abscisic acid (ABA) (Ćalić et al., 2005a), in new studies, lower ABA concentrations were used.

Most reports on the application of $\mathrm{ABA}$ for embryo maturation in woody species refer to species such as Abies (Salajová et al., 1996), Aesculus hyppocastanum (Calić-Dragosavac et al., 2010a; Capuana and Debergh, 1997), Castanea dentata (Robichaud et al., 2004), Coffea canephora (Ducos et al., 2007), Cinnamomum camphora (Shi et al., 2009), and Pterocarpus marsupium (Husain et al., 2010).

The aim of this research was to study the effect of ABA on SSE in horse chestnut microspore and anther culture with the goal of developing a system applicable for the mass production of androgenic embryos with normal morphology.

\section{Materials and Methods}

Plant material and culture conditions. The selected closed flower buds (4 to $5 \mathrm{~mm}$ long) used in the experiments were obtained from a 35-year-old horse chestnut tree (voucher No. P1/16) growing in the Botanical Garden "Jevremovac" of Belgrade University.

Selected buds were disinfected with $95 \%$ ethanol (2 to $3 \mathrm{~min}$ ) and $70 \%$ ethanol ( $5 \mathrm{~min}$ ) followed by three rinses in sterile distilled water. Basal medium (BM) contained Murashige and Skoog (1962) mineral salts, $2 \%$ sucrose, and was supplemented with: panthotehenic acid (10), nicotinic acid (5), vitamin $B_{1}(2)$, adenine sulphate (2), myo-inositol (100), and casein-hydrolysate (200) (mg. $\left.\mathrm{L}^{-1}\right)$. Anther cultures were established on solid induction medium with $0.7 \%$ agar, whereas androgenic embryos were obtained by culturing uninuclear microspores in liquid medium. Solid and liquid induction media contained BM with 2,4-D dichlorphenoxyacetic acid (2,4-D) and kinetin (Kin), $1.0 \mathrm{mg} \cdot \mathrm{L}^{-1}$.

Isolation and culture of microspores. Microspores were isolated from anthers. The anthers were macerated in glass petri dishes using a scalpel. The microspores collected on the surface of the $50-\mu \mathrm{m}$ sieve were carefully washed with liquid induction medium. The microspore suspensions were subcultured every month and refreshed with same medium. The microspore suspensions were kept at $25 \pm 1{ }^{\circ} \mathrm{C}$ in the dark at a density of $4-7 \times$ $10^{5}$ microspores $/ \mathrm{mL}$. Microspore suspension cultures were grown on a horizontal shaker (85 rpm).

Cell suspensions produced after one month were mixed with equal volumes of cooled BM medium $\left(30^{\circ} \mathrm{C}\right)$ and dispensed in petri dishes. The BM medium was supplemented with $0.01 \mathrm{mg} \cdot \mathrm{L}^{-1} 2,4-\mathrm{D}$ and $1 \mathrm{mg} \cdot \mathrm{L}^{-1} \mathrm{Kin}$ and $0.7 \%$ agar.

Isolation and culture of anthers. Seven anthers were inoculated in each culture tube containing $8 \mathrm{~mL}$ of the induction medium. 
Embryo development and multiplication of androgenic embryos from suspension and anther cultures were cultured on medium containing $0.01 \mathrm{mg} \cdot \mathrm{L}^{-1} 2,4-\mathrm{D}$ and $1.0 \mathrm{mg} \cdot \mathrm{L}^{-1}$ Kin. Multiplication embryos were cultured on media for embryo maturation, which was supplemented with filter sterilized ABA.

ABA-free medium $\left(\mathrm{A}_{0}\right)$ was used as a control, and media $\left(A_{1}\right.$ to $\left.A_{6}\right)$ with various concentrations of ABA $(0.01,0.1,0.5,1,2$, and $5 \mathrm{mg} \cdot \mathrm{L}^{-1}$ ) were tested. Subculturing was done every month and the maturation phase lasted a total of three months.

All media were sterilized by autoclaving at $0.9 \times 10^{5} \mathrm{KPa}$ and $114{ }^{\circ} \mathrm{C}$ for $25 \mathrm{~min}$. Androgenic embryos were grown at $25 \pm 1{ }^{\circ} \mathrm{C}$ in conditions of 16/8-h photoperiod (longday ) with irradiance of 33 to $45 \mu \mathrm{mol} \cdot \mathrm{m}^{-2} \cdot \mathrm{s}^{-1}$ produced by cool white fluorescent tubes. Seventy androgenic embryos were cultivated on each medium.

Structural study. Androgenic and secondary somatic embryos were then fixed in formalinacetic acid-ethanol 10:5:85), dehydrated in a graded ethanol series, and embedded in paraffin wax at $58{ }^{\circ} \mathrm{C}$. Sections ( $8 \mu \mathrm{m}$ thick) were stained with hematoxylin and photographed under a Leitz DMRB photomicroscope (Leica, Wetzlar, Germany).

Androgenic and secondary somatic embryos were cut longitudinally and structural investigated. Secondary somatic embryos arose from the subepidermis.

Scanning electron microscopy. The samples were processed for scanning electron microscopy (SEM) without the usual fixation and dehydration procedures. In the SEM studies, the primary androgenic embryos with secondary somatic embryos were placed directly on the stubs and were covered with a thin layer of gold (ion sputtering coating) in a BALETECSCD 005 Sputtering Device, imaging at $15 \mathrm{kV}$, using a JSM-6390 LV (JEOL, Tokyo, Japan) scanning electron microscope. The surface of androgenic and secondary somatic embryos was examined under the SEM and microphotographed.

Statistical analysis. Seventy androgenic embryos per medium $\left(A_{0}\right.$ and $A_{1}$ to $\left.A_{6}\right)$ were analyzed. Three repetitions were performed per each medium. The total number of androgenic embryos analyzed for each studied medium was 210 . The frequency of secondary somatic embryogenesis and the number of secondary somatic embryos were recorded after one month of treatment. For all variables recorded in the measurements, analyses of variance were conducted to test the differences. Statistical analyses were done using Tukey's test at $P \leq 0.05$.

\section{Results}

Visual, scanning, and structural observation. Androgenic embryo differentiation in microspore and anther cultures was rapid and after 4 weeks, the first globular embryos were observed followed by globular, heart-like, torpedo, and cotyledonary embryos. However, cotyledonary embryos showed high variability in morphology (embryos with fused cotyledons with suppressed cotyledon or hypocotyl development) on hormone-free medium. ABA added in media had a positive effect on embryo morphology. Therefore, morphology of ABA-treated embryos was better than ABA non-treated embryos (Fig. 1A-B).

Secondary somatic embryos arose from the subepidermis of primary androgenic embryos.

Visual, scanning, and structural observation confirmed persistence of secondary somatic embryos on cotyledons (Figs. 2A, 2C, and $2 \mathrm{E}$ ) and roots (Figs. 2B, 2D, and 2F) on primary androgenic embryos.

Effect of embryo development stage on secondary somatic embryogenesis. Development stage of primary embryos had a significant effect on secondary embryogenesis. Globular and heart stage showed significantly higher potential for secondary embryogenesis compared with torpedo and cotyledonary embryos on ABA-free medium (Fig. 3). After 4 weeks in microspore culture, globular embryos showed the highest percentage of secondary embryogenesis (99\%) and number of secondary embryos (75) followed by heart [93\% (67)] and torpedo [86\% (57)]. Cotyledonary embryos were poor in their ability to induce secondary embryogenesis with very low percent response $(45 \%)$ as well as number of secondary embryos (27) on ABA-free medium (Figs. 3 and 4).
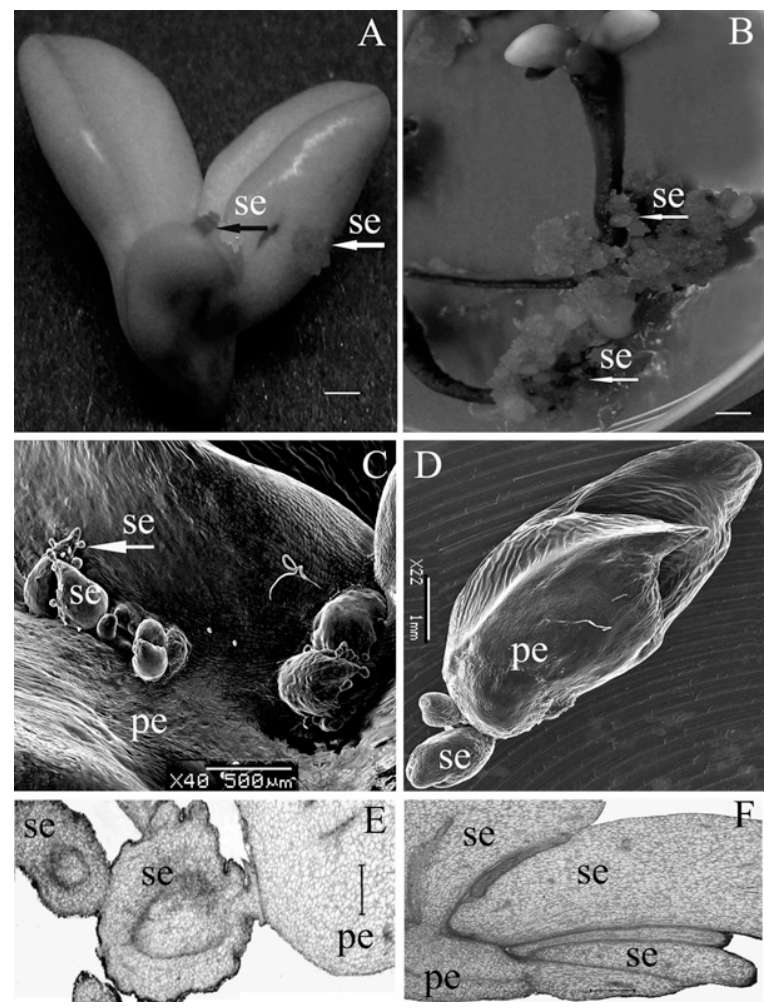

Fig. 2. (A-G) Secondary somatic embryogenesis of horse chestnut. Secondary somatic embryos (se-arrow) on cotyledon $(\mathbf{A})$ and radiculi $(\mathbf{B})$ of androgenic embryos. Bars $=1 \mathrm{~mm} ; 10 \mathrm{~mm}$. (C) Scanning electron micrograph of secondary somatic embryos (se-arrow) on cotyledon and radiculi (D) of primary androgenic embryos (pe). Bars $=500 \mu \mathrm{m} ; 1 \mathrm{~mm}$. (E) Histological section (LS) of a primary androgenic (pe) embryo with two generations of secondary somatic embryos at different stages, originating from the tissue at cotyledon. Bar $=400 \mu \mathrm{m}$. (F) Histological section (LS) of primary androgenic embryo (pe) showing emergence of a secondary somatic embryos at different stages, originating from the tissue at the root pole. $\mathrm{Bar}=400 \mu \mathrm{m}$. 
Cotyledonary embryos induced secondary embryogenesis with a very low response [22\% (16)] on ABA-free medium (Figs. 3 and 4$)$.

Abscisic acid effect on secondary somatic embryogenesis. The highest number of secondary somatic embryos was formed on ABA-free medium (Fig. 5). The number of secondary somatic embryos was three times higher on ABA-free medium than on $\mathrm{A}_{1}$ medium enriched with $0.01 \mathrm{mg} \cdot \mathrm{L}^{-1}$ ABA. With a decreasing ABA concentration from $5 \mathrm{mg} \cdot \mathrm{L}^{-1}$ to $0.01 \mathrm{mg} \cdot \mathrm{L}^{-1}$, the number of secondary somatic embryos increased approximately eight times (Fig. 5). The optimal ABA concentration for secondary somatic embryo induction and production of high-quality embryos was $0.01 \mathrm{mg} \cdot \mathrm{L}^{-1}$.

\section{Discussion}

ABA is commonly used at various ( 0.2 to $20 \mathrm{mg} \cdot \mathrm{L}^{-1}$ ) concentrations to improve somatic embryo development of the horse chestnut (Ćalić-Dragosavac et al., 2010a; Capuana and Debergh, 1997), American chestnut (Robichaud et al., 2004), fir species (Salajová et al., 1996), camphor tree (Shi et al., 2009), Indian kino tree (Husain et al., 2010), and other species. Low ABA concentrations improved production in two different ways: 1) by increasing somatic embryo number; and 2) by enhancing embryo quality.

Our results that the morphology of ABAtreated horse chestnut embryos were better than non-treated embryos is in agreement with the results reported by Capuana and Debergh (1997).

Globular and torpedo embryos had two or more times higher embryogenic potential than cotyledonary embryos. This result can be explained by the fact that the globular embryos are less differentiated than cotyledonary embryos, meaning that they are more totipotent.

Our results show that a greater number of secondary somatic embryos are produced in an earlier phase of development with a lower number of embryos. These results are in agreement with the results obtained for red chestnut (Zdravković-Korać et al., 2008).

Furthermore, our findings that secondary somatic embryos develop from subepidermal cotyledon cells and the hypocotyl of horse chestnut and that the largest number secondary embryos form on hormone-free medium is consistent with the findings of FernándezGuijarro et al., 1995 and Radojević (1995). A tested horse chestnut genotype had higher embryo induction and secondary somatic embryo production in microspore culture than anther culture. These results were expected because microspore culture has been found to be five to 10 times more effective than androgenesis induction in anther culture within the same genotype (Ćalić et al., 2003; Höfer, 2004).

Secondary somatic embryogenesis is the basis for clonal mass propagation (Merkle, 1995). Our experiments aimed to evaluate the induction of SSE and plant regeneration of

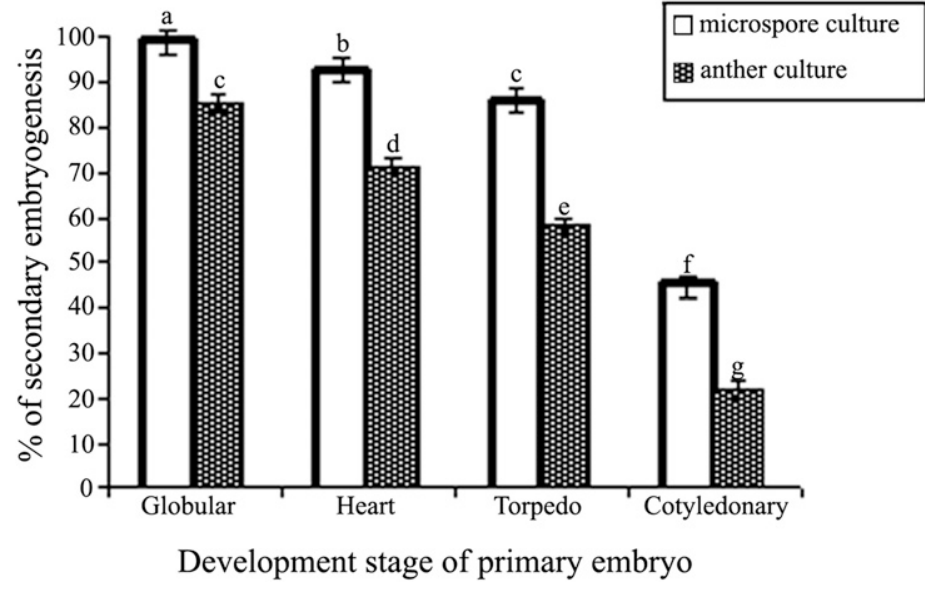

Fig. 3. Influence of development stage of primary horse chestnut embryos in microspore and anther culture on the percentage of secondary somatic embryogenesis on hormone-free medium. Means denoted by the same letter were not significantly different $(P \leq 0.05)$ according to Turkey's test.

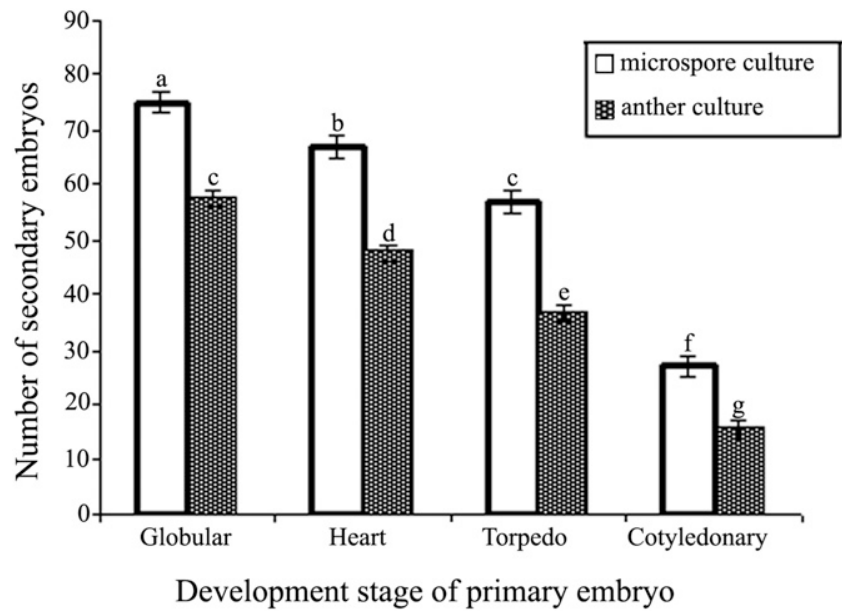

Fig. 4. Influence of development stage of primary horse chestnut embryos in microspore and anther culture on the number of secondary embryos on hormone-free medium. Means denoted by the same letter were not significantly different $(P \leq 0.05)$ according to Tukey's test.

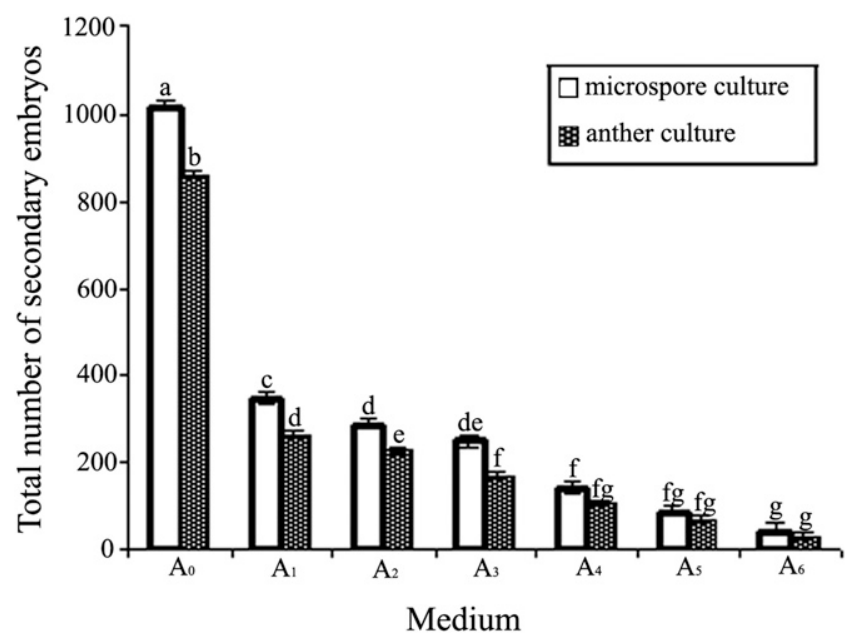

Fig. 5. Effect of ABA on total secondary embryos number in microspore and anther culture. Means denoted by the same letter were not significantly different $(P \leq 0.05)$ according to Tukey's test. ABA $=$ abscisic acid. 
androgenic embryos of $A$. hyppocastanum to develop efficient in vitro regeneration methods to be used in experiments applicable for the silvicultural and pharmaceutical industry.

\section{Literature Cited}

Bombardelli, E., P. Morazzoni, and A. Griffini. 1996. Aesculus hippocastanum L. Fitoterapia 67:483511.

Caldas, E.D. and L.L. Machado. 2004. Cadmium, mercury and lead in medicinal herbs in Brazil. Food Chem. Toxicol. 42:599-603.

Ćalić, D., S. Zdravković-Korać, S. Jevremović, M. Guć-Š́ekić, and Lj. Radojević. 2003. Efficient haploid induction in microspore suspension culture of Aesculus hippocastanum and karyotype analysis. Biol. Plant. 47:289-292.

Ćalić, D., S. Zdravković-Korać, and Lj. Radojević. 2005a. Secondary embryogenesis in androgenic embryo cultures of Aesculus hippocastanum L. Biol. Plant. 49:435-438.

Ćalić, D., S. Zdravković-Korać, and Lj. Radojević. 2005b. Plant regeneration in anther culture of yellow buck (Aesculus flava Marshall.), p. 183185. In: Proc. Final Conference COST 843 and COST 851 Joint Meeting, Stara Lesna, Slovakia.

Ćalić-Dragosavac, D., S. Zdravković-Korać, B. Bohanec, Lj. Radojević, B. Vinterhalter, S. Stevović, A. Cingel, and J. Savić. 2010a. Effect of activated charcoal, abscisic acid and polyethylene glycol on maturation, germination and conversion of Aesculus hippocastanum androgenic embryos. Afr. J. Biotechnol. 9:37863793.

Ćalić-Dragosavac, D., S. Zdravković-Korać, K. Šavikin-Fodulović, Lj. Radojević, and B. Vinterhalter. 2010b. Determination of aescin content in androgenic embryos and hairy root culture of Aesculus hippocastanum (Hippocastanaceae). Pharm. Biol. 48:563-567.

Capuana, M. and P.C. Debergh. 1997. Improvement of the maturation and germination of horse chestnut somatic embryos. Plant Cell Tissue Organ Cult. 48:23-29.

Ducos, J.P., G. Labbe, C. Lambot, and V. Pétiard. 2007. Pilot scale process for the production of pre-germinated somatic embryos of selected robusta (Coffea canephora) clones. In Vitro Cell. Dev. Biol. Plant 43:652-659.

Fernández-Guijarro, B., C. Celestino, and $\mathrm{M}$. Toribio. 1995. Influence of external factors on secondary embryogenesis and germination in somatic embryos from leaves of Quercus suber. Plant Cell Tissue Organ Cult. 41:99-196.

Gilbert, M., S. Guichard, J. Freise, J.C. Grégorie, W. Heitland, N.C. Straw, C. Tilbury, and S. Augustin. 2005. Forecasting Cameraria ohridella invasion dynamics in recently invaded countries: From validation to prediction. J. Appl. Ecol. 42:805-813.

Höfer, M. 2004. In vitro androgenesis in appleImprovement of the induction phase. Plant Cell Rpt. 22:365-370.

Husain, M.K., M. Anis, and A. Shahyad. 2010. Somatic embryogenesis and plant regeneration in Pterocarpus marsupium Roxb. Trees (Berl.) 24:781-787.

Matsuda, H., Y. Li, T. Murakami, K. Ninimiya, N. Araki, M. Yoshikawa, and I. Yamahara. 1997. Effects of aescins Ia, Ib, IIa, IIb from horse chestnut, the seeds of Aesculus hippocastanum on acute inflammation in animals. Biol. Pharm. Bull. 20:1092-1095.

Merkle, S.A. 1995. Strategies for dealing with limitations of somatic embryogenesis in hardwood trees. Plant Tissue Cult. Biotech. 1:112121.
Murashige, T. and F. Skoog. 1962. A revised medium for rapid growth and bioassys with tobacco tissue cultures. Physiol. Plant. 15:473-497.

Radojević, Lj. 1995. Somatic embryogenesis in horse chestnut (Aesculus hippocastanum L.), p. 409-444. In: Jain, S., P. Gupta, and R. Newton (eds.). Somatic embryogenesis in woody plants. Kluwer, Dordrecht, The Netherlands.

Robichaud, R., V. Lessard, and S.A. Merkle. 2004. Treatments affecting maturation and germination of American chestnut somatic embryos. J. Plant Physiol. 161:957-969.

Salajová, T., J. Jásik, A. Kormuták, J. Salaj, and I. Hakman. 1996. Embryogenic culture initiation and somatic embryo development in hybrid firs (Abies alba $\times$ Abies cephalonica, and Abies alba $\times$ Abies numidica). Plant Cell Rpt. 15:527-530.

Shi, X., X. Dai, G. Liu, and M. Bao. 2009. Enhancement of somatic embryogenesis in camphor tree (Cinnamomum camphora L.): Osmotic stress and other factors affecting somatic embryo formation on hormone-free medium. Trees (Berl.) 23:1033-1042.

Wilkinson, J.A. and A.M.G. Brown. 1999. Horse chestnut-Aesculus hippocastanum: Potential applications in cosmetic skin-care products. Intl. J. Cosmet. Sci. 21:437-447.

Yang, X., J. Zhao, Y. Cui, X. Liu, C. Ma, M. Hattori, and L. Zhaung. 1999. Anti HIV-1 protease triterpenoid saponins from the seeds of Aesculus chinensis. J. Nat. Prod. 62:1510-1513.

Zdravković-Korać, S., D. Ćalić-Dragosavac, B. Uzelac, D. Janošević, S. Budimir, B. Vinterhalter, and D. Vinterhalter. 2008. Secondary somatic embryogenesis versus caulogenesis from somatic embryos of Aesculus carnea Hayne: Developmental stage impact. Plant Cell Tissue Organ Cult. 94:225-231. 